ACS Appl Mater Interfaces. 2019 July 10; 11(27): 23919-23925. doi:10.1021/acsami.9b07912.

\title{
Polydopamine-Based Interfacial Engineering of Extracellular Matrix Hydrogels for the Construction and Long-Term Maintenance of Living Three-Dimensional Tissues
}

\author{
Sunghee E. Park ${ }^{\dagger, \|}$, Andrei Georgescu ${ }^{\dagger}, \|$, Jeong Min $\mathrm{Oh}^{\dagger}$, Keon Woo Kwon ${ }^{\dagger}$, Dongeun \\ Huh $^{*}, \dagger, \ddagger, \S$ \\ tDepartment of Bioengineering, University of Pennsylvania, Philadelphia, Pennsylvania 19104, \\ United States \\ \#Institute for Regenerative Medicine, Perelman School of Medicine, University of Pennsylvania, \\ Philadelphia, Pennsylvania 19104, United States \\ §NSF Science and Technology Center for Engineering Mechanobiology, University of \\ Pennsylvania, Philadelphia, Pennsylvania 19104, United States
}

\begin{abstract}
Diverse biological processes in the body rely on the ability of cells to exert contractile forces on their extracellular matrix (ECM). In three-dimensional (3D) cell culture, however, this intrinsic cellular property can cause unregulated contraction of ECM hydrogel scaffolds, leading to a loss of surface anchorage and the resultant structural failure of in vitro tissue constructs. Despite advances in the 3D culture technology, this issue remains a significant challenge in the development and long-term maintenance of physiological 3D in vitro models. Here, we present a simple yet highly effective and accessible solution to this problem. We leveraged a single-step surface functionalization technique based on polydopamine to drastically increase the strength of adhesion between hydrogel scaffolds and cell culture substrates. Our method is compatible with different types of ECM and polymeric surfaces and also permits prolonged shelf storage of functionalized culture substrates. The proof-of-principle of this technique was demonstrated by the stable long-term (1 month) 3D culture of human lung fibroblasts. Furthermore, we showed the robustness and advanced application of the method by constructing a dynamic cell stretching system and performing over 100000 cycles of mechanical loading on 3D multicellular constructs for visualization and quantitative analysis of stretch-induced tissue alignment. Finally, we demonstrated the potential of our technique for the development of microphysiological in vitro models by establishing microfluidic 3D co-culture of vascular endothelial cells and fibroblasts to engineer self-assembled, perfusable 3D microvascular beds.
\end{abstract}

\section{Graphical Abstract}

\footnotetext{
*Corresponding Author huhd@seas.upenn.edu (D.H.).

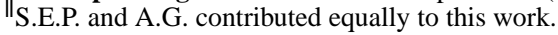

Supporting Information

The Supporting Information is available free of charge on the ACS Publications website at DOI: 10.1021/acsami.9b07912.

Experimental section; hydrogel structure in PDA-treated and untreated PDMS chambers (Figure S1); ECM hydrogel adhesion test

(Figure S2); PDA coating maintenance after shelf storage (Figure S3) (PDF)
} 

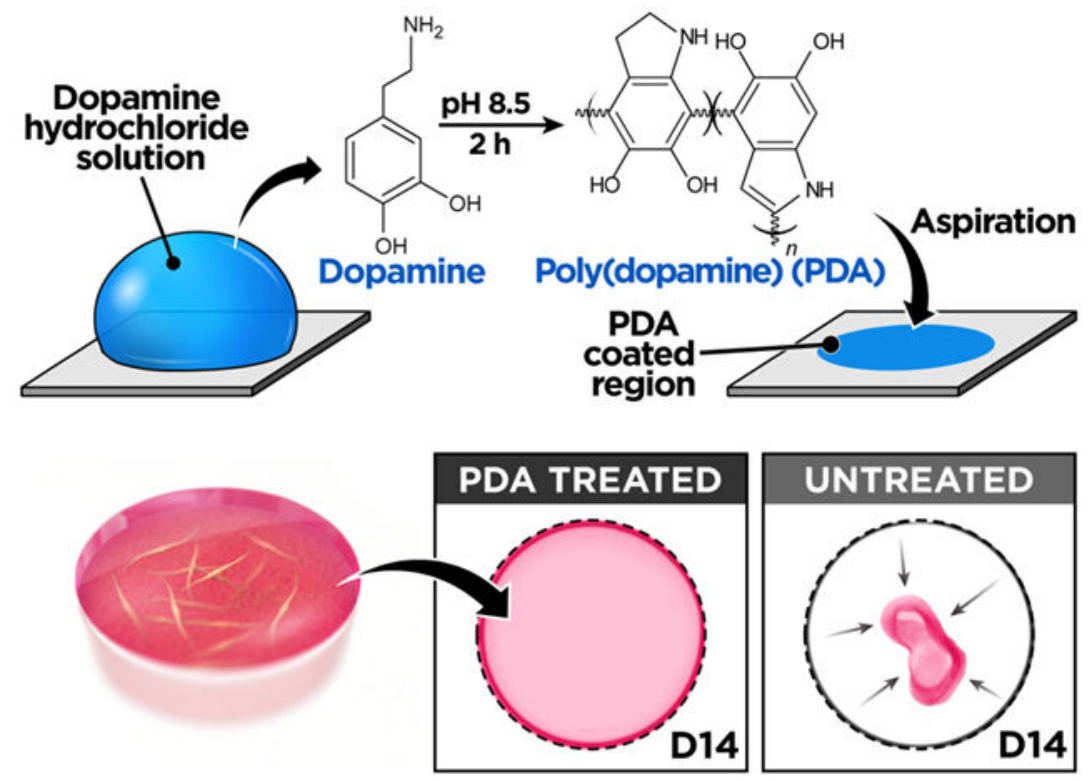

\section{Keywords}

3D culture; hydrogel; polydopamine; microfluidics; organ-on-a-chip

\section{INTRODUCTION}

Growing cells in extracellular matrix (ECM) hydrogels is a common technique to model the native three-dimensional (3D) microenvironment of cells in the body. Substantial evidence has shown that this culture configuration promotes structural and functional differentiation of cells and allows them to express their physiological phenotype in a more efficient manner. ${ }^{1-3}$ Based on these findings, considerable efforts have been made over the last two decades to develop novel in vitro techniques and materials that enable growth, differentiation, and maintenance of cells in physiological 3D environments. ${ }^{4,5}$ Significant progress in this area has led to a wave of 3D culture models designed to address the inherent limitations of traditional two-dimensional (2D) culture, ranging from simple cell-laden hydrogel constructs to bioprinted 3D tissues and microengineered organ-on-a-chip systems. ${ }^{6,7}$

As an essential component of these 3D culture systems, ECM-derived hydrogels provide structural support for cell adhesion and produce insoluble microenvironmental cues that can instruct cultured cells to exhibit their 3D phenotype ${ }^{8,9}$ Despite their desirable properties and important biological function, however, ECM hydrogels also pose unique challenges to in vitro culture. As cells embedded in a hydrogel scaffold attach and spread, they develop intracellular tension due to the contraction of the actomyosin cytoskeleton, which is transmitted through focal adhesions to exert mechanical forces on the surrounding ECM. 10,11 Although these cell-generated traction forces play an essential role in a variety of fundamental biological processes in vivo, ${ }^{12}$ the sustained process of force generation during prolonged culture can cause progressive contraction of highly deformable ECM scaffolds, leading to hydrogel detachment from culture vessels and the resultant shrinkage and collapse 
of 3D tissue constructs in an uncontrolled manner. This type of structural failure has been a major problem in the long-term culture of adherent cells in ECM hydrogels, which is often required for the development of physiologically relevant $3 \mathrm{D}$ in vitro models.

In this paper, we present a simple surface engineering technique to address this problem and demonstrate its application to the construction of advanced 3D culture models. Our method is based on the modification of surface chemistry using poly(dopamine) (PDA), a musselinspired multifunctional material produced by oxidative polymerization or selfassembly of catecholamines in aqueous alkaline solutions. ${ }^{13-15}$ PDA can be deposited spontaneously via a single-step dip coating process to form a thin, biocompatible conformal film on numerous types of organic and inorganic surfaces. ${ }^{13-15}$ Researchers have demonstrated the use of PDA surface coating to achieve strong interfacial adhesion for a wide array of biomedical applications including cell patterning, ${ }^{16,17}$ immobilization of proteins and nanoparticles, ${ }^{18,19}$ biomineralization, ${ }^{20,21}$ and vascular tissue engineering. ${ }^{22,23}$ Inspired by these studies, we investigated whether PDA could be used as a simple approach for the surface functionalization of 3D culture systems to strengthen the anchorage of ECM hydrogel scaffolds during the long-term culture.

\section{RESULTS AND DISCUSSION}

To test the feasibility of this approach, we first conducted a preliminary study to examine the effect of PDA on surface adhesion of acellular ECM hydrogels. Our investigation was carried out using poly(dimethylsiloxane) (PDMS) as a model substrate, which is widely used to engineer advanced 3D cell culture platforms due to its biocompatibility, high gas permeability, ease of fabrication, and optical transparency. ${ }^{24,25}$ First, we prepared a flat slab of PDMS and incubated it in a $2.0 \mathrm{mg} / \mathrm{mL}$ (w/v in $10 \mathrm{mM}$ Tris-HCl buffer, $\mathrm{pH} 8.5$ ) of dopamine hydrochloride solution at room temperature for $2 \mathrm{~h}$ to form a thin layer of PDA on the surface (Figure 1A). This single-step coating process changed surface wettability of PDMS and rendered the substrate hydrophilic, as verified by approximately $50 \%$ reduction in static water contact angle (Figure 1B). Following PDA coating, an array of collagen hydrogel droplets was created on the PDMS surface and incubated in an aqueous buffer solution, while being constantly agitated by a rotating shaker at $100 \mathrm{rpm}$ for $12 \mathrm{~h}$ at room temperature (Figure 1C). Under this dynamic condition, the hydrogel array on the PDAtreated substrate remained intact without showing any indications of gel detachment (Figure 1D). In contrast, the vast majority of collagen droplets on untreated PDMS began to lose their anchorage to the substrate and detach within $2 \mathrm{~h}$ (Figure 1D,E), demonstrating the significant enhancement of hydrogel adhesion due to the surface functionalization by PDA. This method produced similar beneficial effects when the same tests were performed at $37^{\circ} \mathrm{C}$, which represents typical temperature conditions for mammalian cell culture, and when we used matrigel to form hydrogel droplets (Figure 1F).

Based on the promising results of our preliminary study, we then constructed a simple 3D culture model to directly assess the effectiveness of using PDA to prevent cellular contractility-induced detachment of ECM hydrogels. For this study, we used primary human lung fibroblasts as a model cell population representing highly contractile cells that reside in a 3D environment in vivo. To establish the 3D culture, the cells were embedded and grown 
in hydrogel scaffolds made out of type I collagen in circular PDMS wells functionalized with PDA (Figure 1G). During cell culture in this system, the fibroblast-containing collagen hydrogel constructs remained firmly attached to the well surface and retained their shape in both horizontal and vertical directions (Figures $1 \mathrm{H}$ and S1). The structural stability of the hydrogel was maintained over 30 days despite significant amounts of serum (2\%) present in the culture medium (Figure 1H). When the PDMS wells were not pretreated with PDA, however, the failure of hydrogel adhesion occurred within the first 4 days of culture and became more evident with time, eventually leading to complete detachment and the spontaneous collapse of the entire tissue constructs within 10 days (Figure 1H). Importantly, PDA was found to be equally effective for preventing hydrogel detachment in conventional polystyrene well plates (Figure 1I). This adhesive property of PDA was also superior to that of other materials that have been used for similar purposes in cell culture (Figure S2) ${ }^{26}$ Moreover, our study revealed that PDA-coated substrates can be stored in ambient air conditions for as long as 4 weeks without a significant loss of their adhesive properties (Figure S3).

Although further investigation is necessary, the significantly enhanced hydrogel adhesion demonstrated in these experiments may be attributed to covalent bonding of amine and thiol groups in the collagen hydrogel with PDA through Michael addition and Schiff base formation. ${ }^{27,28} \mathrm{PDA}$ can also engage in noncovalent interactions with the ECM hydrogel through hydrogen bonding, cation $-\pi, \pi-\pi$, and electrostatic interactions, ${ }^{29,30}$ which can further increase the strength of interfacial adhesion. The hydrophilicity of PDA-treated PDMS may be another contributing factor, as increased surface wettability can facilitate the interaction of highly hydrated ECM scaffolds with the surface of the culture chamber.

Since the geometry of the culture well is a major determinant of boundary constraints that dictate the spatial distribution of cell-generated contractile forces within the hydrogel scaffold, we also examined the performance of PDA under different stress conditions created by changing the shape of the culture chamber. Unlike the original circular wells, the new chambers were designed to have sharp corners to generate local regions of stress concentration (Figure 2A). Interestingly, this geometrically induced nonuniform mechanical environment had negligible effects on the adhesive function of PDA. Regardless of the chamber shape, the hydrogel constructs cultured in the PDA-coated wells showed no measurable structural changes during the course of 10-day culture tested in this study (Figure 2B). The same culture in the nontreated system, by contrast, resulted in the failure of hydrogel adhesion that initially occurred at the sharp corners of the chambers and propagated along the edges (Figure 2C).

Importantly, the ability to stably maintain cell-laden hydrogel constructs made it possible to probe the morphology and structural organization of embedded cells within their 3D microenvironment during long-term culture. As illustrated by immunofluorescence staining of the actin cytoskeleton (Figure 2D), the fibroblasts cultured in collagen hydrogel for 10 days assumed an elongated morphology and extended dendritic processes into their surrounding matrix. Collectively, these cells were organized into a 3D cellular network in a

The authors declare no competing financial interest. 
randomly oriented manner, which is indicative of the isotropic mechanical microenvironment produced by the spatially uniform adhesion of the circular hydrogel scaffold to the substrate. Culture of other types of contractile cells, such as hepatic stellate (hHSC) and muscle cells (C2C12), gave rise to similar cell morphologies and multicellular organizations (Figure 2E). A loss of boundary constraints due to hydrogel detachment in nontreated wells, however, led to drastic changes in tissue microarchitecture. Regardless of the type, the cells in the collapsed hydrogel constructs lost their 3D morphology and formed highly dense, planar structures in which the morphological characteristics of individual cells were similar to those seen in 2D culture (Figure 2F). Taken together, these results demonstrate that PDA provides a simple yet versatile approach to establish, maintain, and analyze the long-term 3D culture of contractile cells in ECM hydrogel scaffolds.

In the next phase of our study, we explored the feasibility of leveraging our surface engineering technique to build an advanced in vitro platform capable of supporting 3D cell culture in physiologically relevant dynamic mechanical environments. Specifically, we designed a computer-controlled motorized mechanical actuator integrated with an array of PDMS cell culture chambers to subject fibroblasts-laden hydrogel constructs to cyclic stretch to simulate breathing-induced deformation of the lung parenchyma (Figure 3A). As a preliminary study, we first tested the ability of PDA coating to maintain surface tethering of acellular collagen and fibrin hydrogels during prolonged operation of the stretching system. In both cases, PDA-coated wells showed significantly increased interfacial adhesion compared to the untreated group, effectively preventing hydrogel detachment during 86400 cycles of uniaxial stretch at $10 \%$ strain and physiological breathing rates $(0.33 \mathrm{~Hz})$ over 3 days (Figure 3B).

These functionalized chambers were then used to culture primary human lung fibroblasts in collagen hydrogels and to stretch them with $10 \%$ cyclic strain at $0.33 \mathrm{~Hz}$. In response to sustained force application in this system, the vast majority of cells was observed to reorient themselves in the direction of applied strain (Figure 3C,D). After incessant stretching for 5 consecutive days, $88 \%$ of the cells displayed directional elongation and alignment (Figure $3 \mathrm{E}$, middle). Our result is in contrast with the perpendicular reorientation of fibroblasts cultured and cyclically stretched on 2D substrates. ${ }^{31,32}$ The parallel alignment observed in our system, however, has recently been identified as a unique mechanosensitive behavior of fibroblasts in 3D matrices. ${ }^{33,34}$ In comparison to the static culture, the applied strain also significantly increased the number of fibroblasts, presumably due to the promotive effect of mechanical stretch on cell proliferation previously reported in fibroblast cultures (Figure 3E, bottom). ${ }^{35,36}$ Under the same stretching conditions, many of the constructs in the nonfunctionalized replicates detached from the wells within $24 \mathrm{~h}$. The cells in these free-floating hydrogels still exhibited directional elongation, but the unconstrained contraction of the detached scaffolds led to a loss of global tissue alignment, generating multicellular aggregates with highly disorganized microarchitecture (Figure 3C, bottom row).

Studying the behavior of cells in mechanically dynamic organs in vitro often requires complex, customized systems to affix and stabilize cell-laden 3D hydrogel scaffolds undergoing structural deformation, which greatly increases the burden of cell culture experiments. By relying upon PDA coating to create anchored hydrogels in an array format, 
our system offers a simple and accessible means to enable these types of studies with significantly increased throughout. As demonstrated by the stability of our 3D fibroblast constructs subjected to a continuous 144000 strain cycles during the 5-day experiment, hydrogel adhesion mediated by PDA is strong, durable and provides compelling advantages not only for establishing the long-term 3D culture but also for modeling the dynamic characteristics of the native 3D tissue environment.

Finally, we set out to demonstrate the use of PDA for constructing microengineered in vitro models of specialized 3D human tissues. Organs-on-chips and microphysiological systems have garnered considerable attention in recent years due to their potential to serve as more predictive and physiologically relevant alternatives to traditional cell culture and animal models. ${ }^{37}$ By making it possible to control cells and their microenvironment with unprecedented spatiotemporal precision, these microengineered systems provide a powerful platform to mimic and interrogate the complexity of human physiological systems in vitro. 6,38 Early studies in this area have demonstrated the instrumental role of conventional 2D cell culture techniques in creating organ-on-chip models of various tissue types. ${ }^{39-41}$ With the increasing interest in developing more advanced and realistic in vitro models, however, there is a significant need for reliable and accessible approaches to establishing and maintaining 3D cell culture in microfabricated devices. ${ }^{42,43}$ Efforts to meet this emerging need have been complicated by the susceptibility of hydrogel scaffolds to contractive deformation and the resultant loss of surface adhesion during the long-term culture, which tends to be more detrimental in microdevices due to the small size of engineered tissue constructs.

To demonstrate the potential of PDA as an effective solution to this problem, we created a PDMS microfluidic device for co-culture of vascular endothelial cells and fibroblasts in ECM hydrogels to engineer 3D networks of perfusable blood vessels (Figure 4A,B). The key feature of this device was a pair of microfabricated protrusions on the channel ceiling that permitted capillary pinning-based spatial confinement of hydrogel precursor solution in the central region to form a 3D fibrin scaffold encapsulating primary human lung fibroblasts and human umbilical vein endothelial cells (HUVECs) (Figure 4B,C). To provide fluidic access for the delivery of culture media, the cell-laden hydrogel was flanked by two microchannels lined with HUVECs (Figure 4C). Importantly, PDA coating was crucial to sustaining this microengineered 3D culture model. During the course of 28-day culture, the hydrogel scaffolds in the PDA-coated devices remained firmly anchored to the channel surfaces and supported the growth and maintenance of the embedded cells. In particular, the endothelial cells in these constructs underwent spreading and proliferation initially, but soon they began to establish intercellular connections and assemble themselves into 3D network structures (Figure 4C). This process continued for approximately 2 weeks, eventually leading to the formation of 3D microvascular beds composed of interconnected endothelial tubes (Figure 4D). In the absence of PDA coating, similar patterns of endothelial selfassembly were observed in the first 2-3 days. With further progression of culture, however, the hydrogels rapidly lost their anchorage to the channel walls and showed significant contraction, during which the endothelial networks were destroyed and collapsed into dense cellular aggregates (Figure 4E). 
Another important observation was that the engineered vessels assembled and maintained in the PDA-treated channels were perfusable, as visualized by the intravascular flow of fluorescently labeled dextran through the entire vascular bed (Figure 4F). In contrast, gel detachment presumably from the bottom channel surfaces in the uncoated devices created a low-resistance underpass, causing dextran to simply flow beneath the detached gel during perfusion (Figure 4G). These results reiterate the effectiveness of PDA as a chemical linker and highlight its critical role in generating perfusable 3D vascular constructs, which could potentially be combined with other types of cells to engineer in vivo-like vascularized tissues with significantly improved fidelity. Although our study focused on the vasculature as a model system, the same technique is readily applicable to mimicking other types of 3D tissues, suggesting the added value of PDA as a powerful technique for in vitro reproduction of complex 3D tissue architecture and native physiological function in microengineered cell culture devices.

\section{CONCLUSIONS}

In summary, this paper presents a facile yet highly effective and broadly applicable approach to enable the long-term (1 month or longer) 3D cell culture in ECM hydrogel scaffolds. By leveraging the simplicity and versatility of PDA, our method allows for single-step, materialindependent, and low-cost surface functionalization of culture vessels to achieve significantly enhanced strength and stability of hydrogel adhesion in both traditional and advanced 3D in vitro models. The coating procedure requires only two reagents (Tris- $\mathrm{HCl}$ buffer and dopamine hydrochloride) and can be completed within $2 \mathrm{~h}$ simply by immersing culture substrates in a dopamine solution without the need for specialized equipment, making our technique attractive for widespread and routine use in any laboratory setting. PDA coating is also amenable to shelf storage prior to use and, thus, lends itself well to the large-scale batch production and distribution of cell culture substrates and devices for the purposes of research collaboration and commercialization. Moving beyond the proof-ofconcept demonstrations presented here, future efforts to realize the full potential of our technique will require systematic investigation of key coating parameters to identify optimal conditions that maximize the strength, long-term stability, and shelf-life of PDA coating. Research attention should also be paid to the drawbacks of PDA-based coating techniques. For example, recent studies have reported significant increases in the elastic moduli of PDAcoated surfaces, ${ }^{44}$ which may lead to unwanted alterations in the mechanical properties of cell culture substrates. Nonspecific binding of biomolecules (e.g., serum proteins) on PDAtreated substrates is another potential problem that needs to be examined and addressed in future investigations. ${ }^{45}$

In conclusion, we believe that our method will make significant contributions to expanding the application of the 3D culture and developing new types of in vitro models with advanced capabilities to emulate the dynamic and complex behavior of their in vivo counterparts for a variety of biomedical applications.

\section{Supplementary Material}

Refer to Web version on PubMed Central for supplementary material. 


\section{ACKNOWLEDGMENTS}

We thank $\mathrm{G}$. Al and S. Chung for their intellectual input and technical assistance. This work was supported by the National Institutes of Health (NIH) (1DP2HL127720-01, 1UG3TR002198-01, 1UC4DK104196-01), the Paul G. Allen Family Foundation, the Cancer Research Institute, and the University of Pennsylvania. This work was carried out in part at the Singh Center for Nanotechnology, which is supported by the NSF National Nanotechnology Coordinated Infrastructure Program under grant NNCI-1542153. D.H. is a recipient of NIH Director's New Innovator Award and the Cancer Research Institute Technology Impact Award.

\section{REFERENCES}

(1). Pampaloni F; Reynaud EG; Stelzer EHK The Third Dimension Bridges the Gap Between Cell Culture and Live Tissue. Nat. Rev. Mol. Cell Biol 2007, 8, 839-845. [PubMed: 17684528]

(2). Haycock JW 3D Cell Culture: A Review of Current Approaches and Techniques. Methods Mol. Biol 2011, 695, 1-15. [PubMed: 21042962]

(3). Edmondson R; Broglie JJ; Adcock AF; Yang L Three-Dimensional Cell Culture Systems and Their Applications in Drug Discovery and Cell-Based Biosensors. Assay Drug Dev. Technol 2014, 12, 207-218. [PubMed: 24831787]

(4). Fennema E; Rivron N; Rouwkema J; Blitterswijk C; Boer J Spheroid Culture as A Tool for Creating 3D Complex Tissues. Trends Biotechnol 2013, 31, 108-115. [PubMed: 23336996]

(5). Knight E; Przyborski S Advances in 3D Cell Culture Technologies Enabling Tissue-Like Structures to Be Created In Vitro. J. Anat 2015, 227, 746-756. [PubMed: 25411113]

(6). Huh D; Hamilton GA; Ingber DE From 3D Cell Culture to Organs-On-Chips. Trends Cell Biol 2011, 21, 745-754. [PubMed: 22033488]

(7). Murphy SV; Atala A 3D Bioprinting of Tissues and Organs. Nat. Biotechnol 2014, 32, 773-785. [PubMed: 25093879]

(8). Tibbitt MW; Anseth KS Hydrogels as Extracellular Matrix Mimics for 3D Cell Culture. Biotechnol. Bioeng 2009, 103, 655-663. [PubMed: 19472329]

(9). Geckil H; Xu F; Zhang X; Moon S; Demirci U Engineering Hydrogels as Extracellular Matrix Mimics. Nanomedicine 2010, 5, 469-484. [PubMed: 20394538]

(10). Maruthamuthu V; Sabass B; Schwarz US; Gardel ML Cell-ECM Traction Force Modulates Endogenous Tension at Cell-Cell Contacts. Proc. Natl. Acad. Sci. U.S.A 2011, 108, 4708-4713. [PubMed: 21383129]

(11). Schwarz US; Gardel ML United We Stand: Integrating the Actin Cytoskeleton and Cell-Matrix Adhesions in Cellular Mechano-transduction. J. Cell Sci 2012, 125, 3051-3060. [PubMed: 22797913]

(12). Ananthakrishnan R; Ehrlicher A The Forces Behind Cell Movement. Int. J. Biol. Sci 2007, 3, 303-317. [PubMed: 17589565]

(13). Lee H; Dellatore SM; Miller WM; Messersmith PB Mussel-Inspired Surface Chemistry for Multifunctional Castings. Science 2007, 318, 426-430. [PubMed: 17947576]

(14). Ryu JH; Messersmith PB; Lee H Polydopamine Surface Chemistry: A Decade of Discovery. ACS Appl. Mater. Interfaces 2018, 10, 7523-7540. [PubMed: 29465221]

(15). Schanze KS; Lee H; Messersmith PB Ten Years of Polydopamine: Current Status and Future Directions. ACS Appl. Mater. Interfaces 2018, 10, 7521-7522. [PubMed: 29510631]

(16). Sun K; Xie Y; Ye D; Zhao Y; Cui Y; Long F; Zhang W; Jiang X Mussel-Inspired Anchoring for Patterning Cells Using Polydopamine. Langmuir 2012, 28, 2131-2136. [PubMed: 22085048]

(17). Chien H; Kuo W; Wang M; Tsai S; Tsai W Tunable Micropatterned Substrates Based on Poly(Dopamine) Deposition Via Microcontact Printing. Langmuir 2012, 28, 5775-5782. [PubMed: 22397599]

(18). Lee YB; Shin YM; Lee JH; Jun I; Kang JK; Park JC; Shin H Polydopamine-Mediated Immobilization of Multiple Bioactive Molecules for the Development of Functional Vascular Graft Materials. Biomaterials 2012, 33, 8343-8352. [PubMed: 22917738] 
(19). Cong Y; Xia T; Zou M; Li Z; Peng B; Guo D; Deng Z Mussel-Inspired Polydopamine Coating as a Versatile Platform for Synthesizing Polystyrene/Ag Nanocomposite Particles with Enhanced Antibacterial Activities. J. Mater. Chem. B 2014, 2, 3450-3461.

(20). Ryu J; Ku SH; Lee H; Park CB Mussel-Inspired Polydopamine Coating as a Universal Route to Hydroxyapatite Crystallization. Adv. Funct. Mater 2010, 20, 2132-2139.

(21). Xie J; Zhoung S; Ma B; Shuler FD; Lim CT Controlled Biomineralization of Electrospun Poly(ECaprolactone) Fibers to Enhance Their Mechanical Properties. Acta Biomater 2013, 9, 56985707. [PubMed: 23131385]

(22). Ku SH; Park CB Human Endothelial Cell Growth on Mussel-Inspired Nanofiber Scaffold for Vascular Tissue Engineering. Biomaterials 2010, 31, 9431-9437. [PubMed: 20880578]

(23). Ding Y; Yang Z; Bi CW; Yang M; Zhang J; Xu SL; Lu X; Huang N; Huang P; Leng Y Modulation of Protein Adsorption, Vascular Cell Selectivity and Platelet Adhesion by MusselInspired Surface Functionalization. J. Mater. Chem. B 2014, 2, 3819-3829.

(24). Duffy DC; McDonald JC; Schueller OJA; Whitesides GM Rapid Prototyping of Microfluidic Systems in Poly(Dimethylsiloxane). Anal. Chem 1998, 70, 4974-4984. [PubMed: 21644679]

(25). McDonald JC; Whitesides GM Poly(Dimethylsiloxane) as a Material for Fabricating Microfluidic Devices. Acc. Chem. Res 2002, 35, 491-499. [PubMed: 12118988]

(26). Wang L; Sun B; Ziemer KS; Barabino GA; Carrier RL Chemical and Physical Modifications to Poly(Dimethylsiloxane) Surfaces Affect Adhesion of Caco-2 Cells. J. Biomed. Mater. Res., Part A 2010, 93, 1260-1271.

(27). Liu Y; Ai K; Lu L Polydopamine and Its Derivative Materials: Synthesis and Promising Applications in Energy, Environmental, and Biomedical Fields. Chem. Rev 2014, 114, 50575115. [PubMed: 24517847]

(28). Ho CC; Ding S Structure, Properties and Applications of Mussel-Inspired Polydopamine. J. Biomed. Nanotechnol 2014, 10, 3063-3084. [PubMed: 25992429]

(29). Yu M; Hwang J; Deming TJ Role of L-3,4-Dihydroxyphenylalanine in Mussel Adhesive Proteins. J. Am. Chem. Soc 1999, 121, 5825-5826.

(30). Lu Q; Oh DX; Lee Y; Jho Y; Hwang DS; Zeng H Nanomechanics of Cation- $\pi$ Interactions in Aqueous Solution. Angew. Chem 2013, 125, 4036-4040.

(31). Eastwood MV; Mudera C; McGrouther DA; Brown RA Effect of Precise Mechanical Loading on Fibroblast Populated Collagen Lattices: Morphological Changes. Cell Motil. Cytoskeleton 1998, 40, 13-21. [PubMed: 9605968]

(32). Henshaw DR; Attia E; Bhargava M; Hannafin JA Canine ACL Fibroblast Integrin Expression and Cell Alignment in Response to Cyclic Tensile Strain in Three-Dimensional Collagen Gels. J. Orthop. Res 2006, 24, 481-490. [PubMed: 16453340]

(33). Riehl BD; Park JH; Kwon IK; Lim JY Mechanical Stretching for Tissue Engineering: TwoDimensional and Three-Dimensional Constructs. Tissue Eng., Part B 2012, 18, 288-300.

(34). Au-Yeung KL; Sze KY; Sham MH; Chan BP Development of a Micromanipulator-Based Loading Device for Mechanoregulation Study of Human Mesenchymal Stem Cells in ThreeDimensional Collagen Constructs. Tissue Eng., Part C 2010, 16, 93-107.

(35). Sun L; Qu L; Zhu R; Li H; Xue Y; Liu X; Fan J; Fan H Effects of Mechanical Stretch on Cell Proliferation and Matrix Formation of Mesenchymal Stem Cell and Anterior Cruciate Ligament Fibroblast. Stem Cells Int 2016, 2016, 1-10.

(36). Yang G; Crawford RC; Wang JH Proliferation and Collagen Production of Human Patellar Tendon Fibroblasts in Response to Cyclic Uniaxial Stretching in Serum-Free Conditions. J. Biomech 2004, 37, 1543-1550. [PubMed: 15336929]

(37). Bhatia SN; Ingber DE Microfluidic Organs-On-Chips. Nat. Biotechnol 2014, 32, 760-772. [PubMed: 25093883]

(38). Esch EW; Bahinski A; Huh D Organs-On-Chips at the Frontiers of Drug Discovery. Nat. Rev. Drug Discovery 2015, 14, 248-260. [PubMed: 25792263]

(39). Huh D; Matthews BD; Mammoto A; Montoya-Zavala M; Hsin HY; Ingber DE Reconstituting Organ-Level Lung Functions on a Chip. Science 2010, 328, 1662-1668. [PubMed: 20576885] 
(40). Grosberg A; Alford PW; McCain ML; Parker KK Ensembles of Engineered Cardiac Tissues for Physiological and Pharmacological Study: Heart on a Chip. Lab Chip 2011, 11, 4165-4173. [PubMed: 22072288]

(41). Wilmer MJ; Ng CP; Lanz HL; Vulto P; Suter-Dick L; Masereeuw R Kidney-on-a-Chip Technology for Drug-Induced Nephrotoxicity Screening. Trends Biotechnol 2016, 34, 156-170. [PubMed: 26708346]

(42). Low LA; Tagle DA Organs-on-Chips: Progress, Challenges, and Future Directions. Exp. Biol. Med 2017, 242, 1573-1578.

(43). Watson DE; Hunziker R; Wikswo JP Fitting Tissue Chips and Microphysiological Systems into the Grand Scheme of Medicine, Biology, Pharmacology, and Toxicology. Exp. Biol. Med 2017, 242, 1559-1572.

(44). Klosterman L; Ahmad Z; Viswanathan V; Bettinger CJ Synthesis and Measurement of Cohesive Mechanics in Polydopamine Nanomembranes. Adv. Mater. Interfaces 2017, 4, No. 1700041.

(45). Ding YH; Floren M; Tan W Mussel-inspired polydopamine for bio-surface functionalization. Biosurf. Biotribol 2016, 2, 121-136. [PubMed: 29888337] 
A

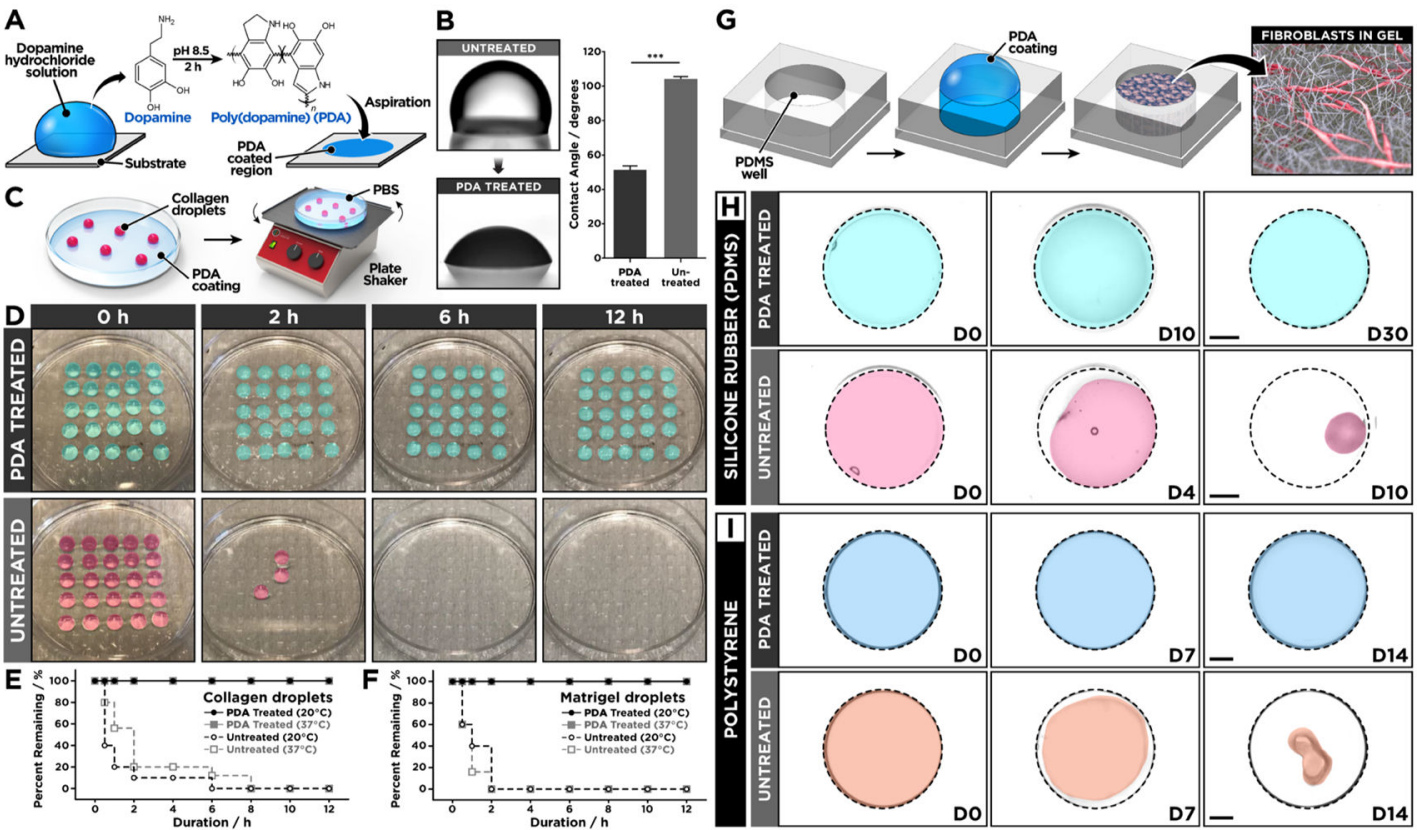

Figure 1.

(A) Surface modification of PDMS is achieved simply by incubating the surface with a dopamine hydrochloride solution to form a thin surface coating. (B) PDA-functionalized PDMS surface shows decreased water contact angle as compared to untreated PDMS. (C) The effect of PDA coating was tested by shaking hydrogel droplets formed on PDA-treated or untreated PDMS surfaces that were immersed in phosphate-buffered saline to prevent dehydration. (D) The hydrogel droplets on the PDA-treated PDMS remain stable for $12 \mathrm{~h}$ of constant agitation, whereas the droplets rapidly detach from the untreated PDMS surface. The quantification of remaining $(\mathrm{E})$ collagen and $(\mathrm{F})$ matrigel droplets at different time points shows significantly improved hydrogel adhesion due to PDA surface coating. $(\mathrm{G}, \mathrm{H})$ Human lung fibroblasts (hLFs) cultured in type I collagen hydrogel remain attached to PDAtreated PDMS for prolonged periods but begin to detach from untreated PDMS wells after 4 days. The hydrogel constructs were pseudo-colored in the presented images. The dotted lines indicate the outlines of culture wells. Scale bars: $2 \mathrm{~mm}$. (I) Similar beneficial effects of PDA are observed in the 3D culture of hLFs in polystyrene wells. Scale bars: $2 \mathrm{~mm}$. *** $P<$ 0.001. Data are expressed as mean $\pm \operatorname{SEM}(n=6)$. 

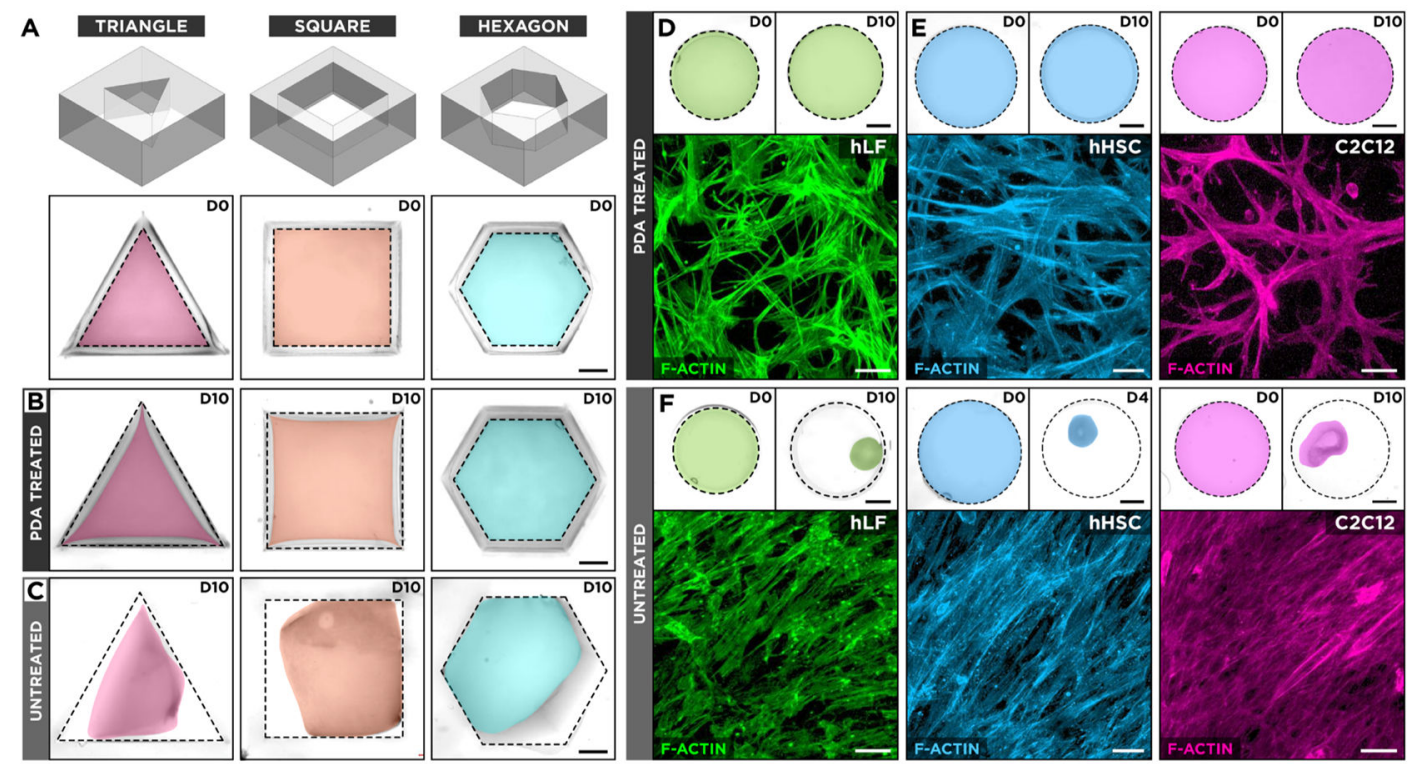

Figure 2.

(A) Effect of geometrical boundary constraints on hydrogel adhesion was assessed by culturing hLFs in type I collagen hydrogel scaffolds in triangular, square, and hexagonal PDMS wells. D0 represents day 0 in culture. (B) The hLF-laden hydrogel constructs in PDA-treated wells retain their surface anchorage, whereas (C) the same culture in untreated counterparts leads to hydrogel detachment and shrinkage. The images show pseudo-colored hydrogels after 10 days of culture (D10). The dotted lines depict the outlines of culture wells. Scale bars: $2 \mathrm{~mm}$. (D, E) Contractile cells grown in surface-bound collagen hydrogels in PDA-treated circular wells extend cellular processes into their surrounding environment and form randomly oriented cellular networks. hHSC represents human hepatic stellate cells. Scale bars: top row: $2 \mathrm{~mm}$, bottom row: $50 \mu \mathrm{m}$. (F) Detachment and unconstrained shrinkage of ECM scaffolds in untreated wells result in the formation of highly dense tissue constructs with directionally elongated microarchitecture. Scale bars; top row: $2 \mathrm{~mm}$, bottom row: 50 $\mu \mathrm{m}$. 

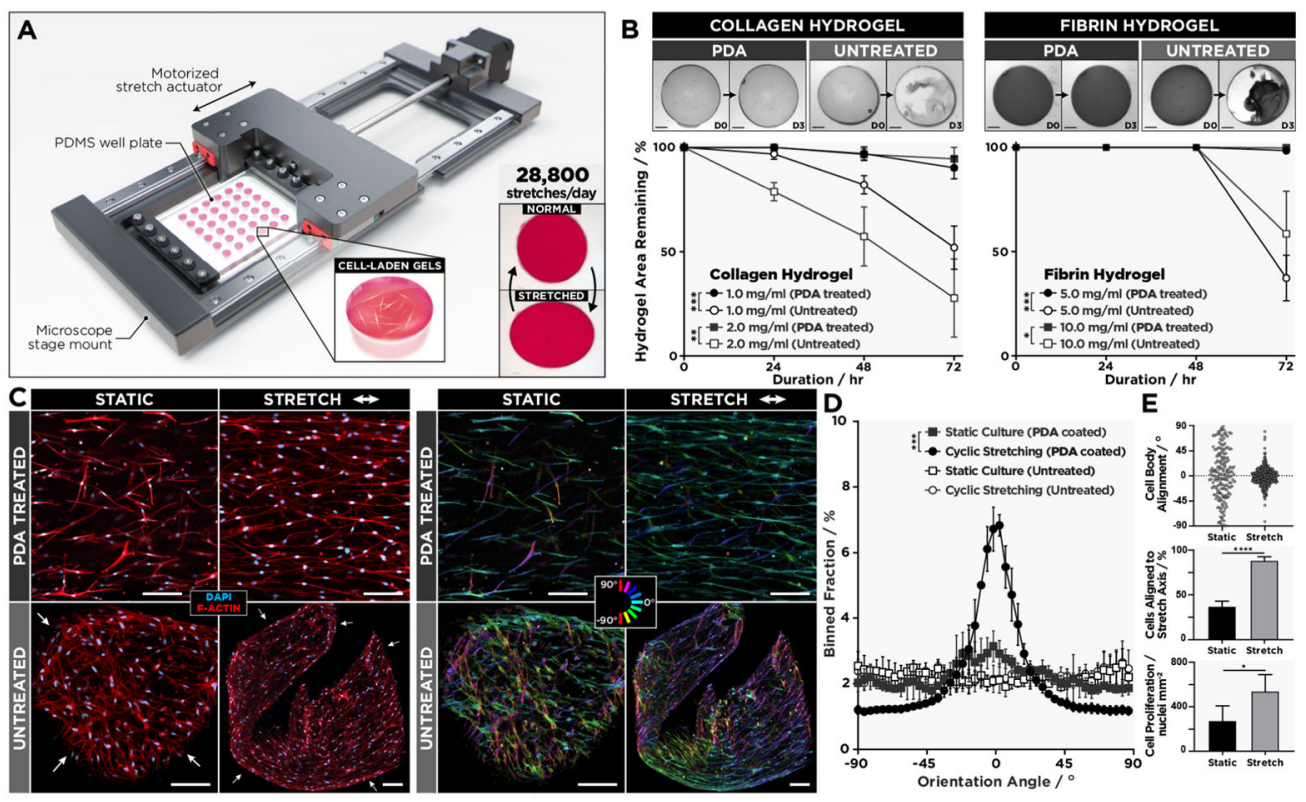

Figure 3.

(A) Schematic of the motorized actuator used for cyclic stretching of cell-laden hydrogel constructs generated in PDMS wells. (B) Adhesion of type I collagen and fibrin gels continuously stretched at $0.33 \mathrm{~Hz}$ for 3 days, which is equivalent to a total of 86400 stretch cycles. Scale bars: $1.5 \mathrm{~mm}$. Data are presented as mean $\pm \mathrm{SD}(n=3)$. (C) As demonstrated by confocal micrographs (a set of four images on the left), hLFs in $2.0 \mathrm{mg} / \mathrm{mL}$ collagen hydrogels firmly anchored to PDA-treated PDMS respond to cyclic stretching at $0.33 \mathrm{~Hz}$ for 5 days (144 000 cycles total) and align in the direction of stretching (top images). This mechanically induced morphological change does not occur in the detached tissue constructs (bottom images). The image set at right shows color-coded orientation maps from directionality analysis where $0^{\circ}$ indicates a horizontal axis parallel to the direction of stretch. Scale bars: $50 \mu \mathrm{m}$. (D) The comparison of cell alignment in cyclically strained and statically cultured devices with and without PDA coating. Data are shown as mean $\pm \operatorname{SD}(n=4)$. (E) In the PDA-coated chambers, stretching induces significant increases in the fraction of aligned cells (top, middle) and the density of cells (bottom). $* P<0.05$; ** $P<0.01 * * * P<$ $0.001 ; * * * * P<0.0001$. 

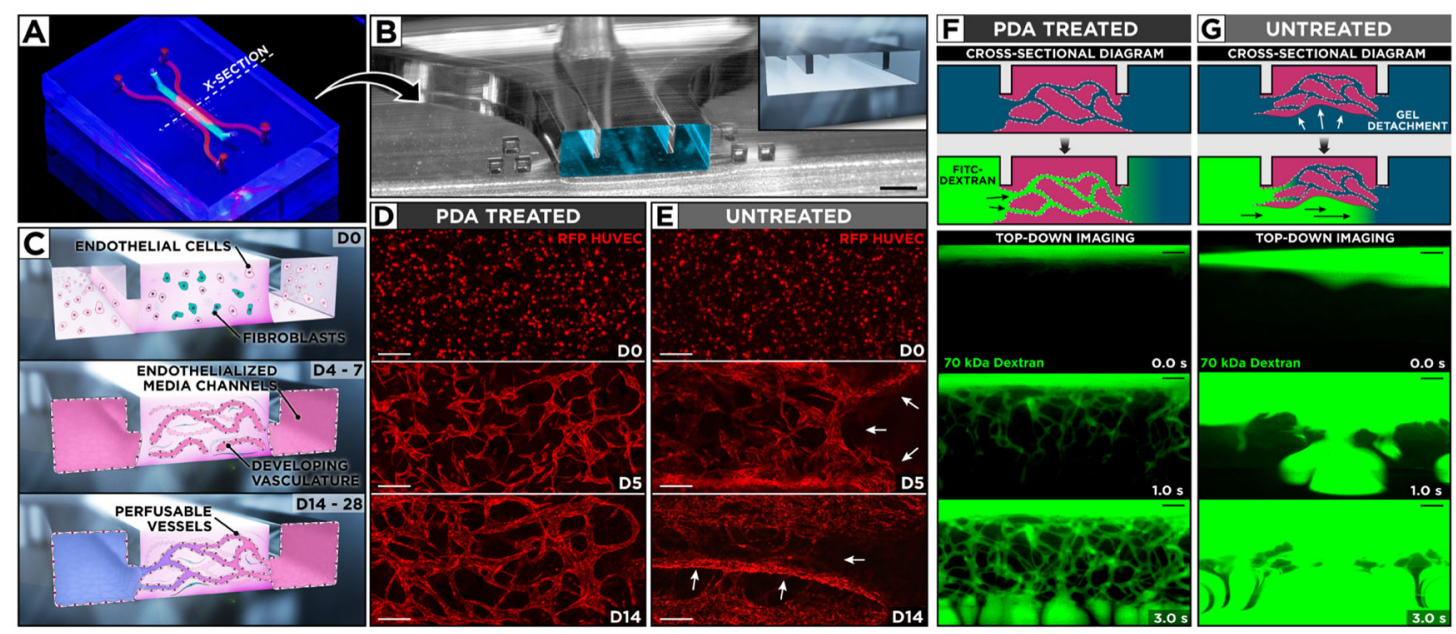

Figure 4.

(A) Photograph of our microengineered device for generation and extended culture of perfusable human blood vessels. (B) The microchannel cross-section along the dotted line in (A) shows the protruding barriers designed to pin hydrogel infused into the central channel. Scale bar: $500 \mu \mathrm{m}$. (C) Vascular endothelial cells and fibroblasts are co-cultured in a central hydrogel scaffold created by capillary pinning between two endothelialized media channels for over 14 days to form a 3D network of self-assembled and perfusable blood vessels lined with living endothelial cells. (D, E) Confocal micrographs of vascular development during 14-day culture of red fluorescent protein-expressing HUVECs and human lung fibroblasts. PDA treatment of the microchannels yields stable hydrogels with well-defined 3D vasculature, whereas untreated devices suffer from hydrogel collapse and the resultant loss of vascular architecture. White arrows indicate areas of gel detachment. Scale bars: $200 \mu \mathrm{m}$. (F) In PDA-coated devices, the engineered vessels become perfusable as demonstrated by the flow of $70 \mathrm{kDa}$ FITC-dextran through their intraluminal compartments. (G) In contrast, the injected fluorescent dye flows beneath and around cell-laden scaffolds in untreated devices as a result of gel detachment. Scale bars: $200 \mu \mathrm{m}$. 